\title{
Evaluación Formativa y Compartida en la Formación Inicial del Profesorado de Educación Física: Resultados tras Cuatro Años de Implementación en una Universidad Pública Chilena
}

\author{
Formative and Shared Assessment in the Initial Training of \\ Physical Education Teachers: Results after Four Years of \\ Implementation in a Chilean Public University
}

\author{
Francisco Javier Gallardo-Fuentes *1 \\ Bastian Carter-Thuillier ${ }^{2}$ \\ Víctor M. López-Pastor ${ }^{3}$ \\ ${ }^{1}$ Universidad de Los Lagos, Osorno, Chile \\ ${ }^{2}$ Universidad Católica de Temuco, Temuco, Chile \\ ${ }^{3}$ Universidad de Valladolid, Segovia, España
}

\begin{abstract}
La transición desde un modelo de enseñanza tradicional a uno competencial ha supuesto un gran cambio de paradigma en la formación universitaria chilena. Trabajar con el alumnado como centro del proceso de Enseñanza-Aprendizaje (E-A) obliga a replantear muchos elementos que intervienen en dichos procesos, especialmente la Evaluación. El objetivo del estudio es conocer la percepción del alumnado tras la aplicación de sistemas de Evaluación Formativa y Compartida en cuatro asignaturas de una universidad chilena. Se llevó a cabo bajo un enfoque cuantitativo en una muestra de 111 estudiantes de Formación Inicial del Profesorado en Educación Física, que participó de cuatro asignaturas que utilizaron sistemas de Evaluación Formativa y Compartida durante cuatro años seguidos. Son las siguientes: curso 2014, asignatura "prácticum" (Edad 24.1 \pm 1.8); curso 2015, asignatura “Actividad Física y Salud para Grupos Especiales” (Edad $22 \pm 2.2)$; curso 2016, asignatura "Gasto Energético y Antropometría” (Edad $23 \pm$ 1.9); y, curso 2017 , asignatura "Gasto Energético y Antropometría” (Edad 23.2 — 2.1). Los principales resultados muestran altas valoraciones a los ítems correspondientes a "ventajas del sistema de evaluación” y bajas valoraciones a los ítems que se presentan como reales inconvenientes de un sistema de evaluación. Se pudo apreciar una alta valoración a los sistemas de evaluación utilizados, así como la presencia de elementos de Evaluación Formativa y Compartida en la Formación Inicial del Profesorado de la universidad en la que se realizó el estudio.
\end{abstract}

Palabras clave: Evaluación formativa; Evaluación compartida; Formación inicial del profesorado; Auto-percepción de competencias; Universidad chilena; Participación del alumnado.

The transition from a traditional model to a competency model has meant a great paradigm shift in Chilean university education. Replace the teaching staff with the students at the center of the Teaching-Learning (E-A) process forces us to rethink many elements that intervene in such processes, including assessment. The objective of the study is to know the perception of the students after the application of Formative and shared Assessment in four subjects from a Chilean university. It was carried out under a quantitative approach in a sample of 111 students (23.2 \pm 2.1$)$, the sample corresponded to students of Initial Teacher Training in Physical

*Contacto: fgallardo@ulagos.cl

issn: 1989-0397

www.rinace.net/riee/

https://revistas.uam.es/riee
Recibido: $\quad 22$ de enero de 2019

$1^{\text {a }}$ Evaluación: 25 de febrero de 2019

$2^{\text {a }}$ Evaluación: 18 de marzo de 2019

Aceptado: $\quad 08$ de abril de 2019 
Education who participated in four systems of Formative and shared Assessment implemented in 2014 in the "practicum" (Age 24.1 \pm 1.8); 2015 in the subject "Physical Activity and Health for Special Groups" (Age $22 \pm 2.2$ ); 2016 in the subject "Energy Expenditure and Anthropometry" (Age $23 \pm 1.9$ ) and 2017 in the subject "Energy Expenditure and Anthropometry" (Age $23.2 \pm 2.1$ ). The main results show high valuated to the items corresponding to "advantages of the Assessment system" and low valuated to the items that are presented as real inconvenient of an Assessment system. It was possible to appreciate a high valuation to the Assessment systems used, as well as the presence of elements of formative and shared Assessment in the Pre-service Physical Education Training of the university where the study was carried out.

Keywords: Formative assessment; Co-Assessment; Pre-service teacher education; Skills self-perception; Chilean university; Student involvement.

\section{Introducción}

Las estructuras formativas universitarias del Espacio Chileno de Educación Superior (ECES) se han adscrito a modelos por competencias, experimentado un claro cambio de paradigma respecto a los modelos tradicionales. Lo anterior ha generado trasformaciones importantes en los escenarios educativos nacionales, puesto que los modelos competenciales estipulan que se debe ubicar en el centro del proceso EnseñanzaAprendizaje (E-A) al alumnado, y está sola intención advierte una brecha importante respecto al enfoque tradicional que reservaba dicho sitial a la figura del profesor (CabalínSilva y Navarro-Hernández, 2008; Tejada, 2009). Por su parte el modelo tradicional enfrenta en el último tiempo múltiples críticas, puesto que habitualmente transforma el proceso de E-A en una instancia de instrucción unidireccional (Ayala, Messing, Labbe y Obando, 2010).

Los modelos competenciales, además, de incorporar al alumnado en el proceso de E-A, buscan una interacción disciplinar, alejándose de la enseñanza de conocimientos aislados que no dialogan con otras disciplinas que integran el currículo. Según Cano (2008), las competencias: "incluyen conjuntos de conocimientos, habilidades y actitudes de carácter muy diferente, incorporando talentos o inteligencias que tradicionalmente desde los sistemas educativos reglados no se habían tenido presentes" (p. 3), es así como estas estructuras curriculares (competenciales), se alejan de "las clásicas divisiones entre asignaturas o las clasificaciones de los saberes que parecen no servir en el contexto actual" (Cano, 2008, pp. 2-3).

Pero si el modelo curricular cambia, también deben hacerlo otros elementos que influyen directamente en el logro del objetivo que el proceso de E-A persigue. Uno de estos elementos es "La Evaluación", autores como Gibbs (2003) la consignan como un arma poderosa a disposición del profesorado, con el potencial de influir significativamente en el modo que el alumnado orienta su aprendizaje, siempre que se utilice de forma adecuada.

Tras la búsqueda de experiencias evaluativas que reportaran ventajas y que incorporaran al alumnado como actor principal del proceso, hemos encontrado numerosas referencias de la propuesta denominada: "Evaluación Formativa y Compartida" (EFyC) (Castejón, LópezPastor, Julián y Zaragoza, 2011; Gallardo-Fuentes y Carter-Thuillier, 2016; GallardoFuentes, López-Pastor y Carter-Tuhillier, 2018; González-Calvo, Martínez-Álvarez, 2018; Hernandez-Elizondo y Salicetti-Fonseca, 2018; López-Pastor, Pérez, Barba y Lorente, 
2016; Martín, 2007). La EFyC es "todo proceso de evaluación cuya finalidad principal es mejorar los procesos de enseñanza-aprendizaje que tienen lugar. Es todo proceso de evaluación que sirve para que el alumnado aprenda más (y/o corrija sus errores) y para que el profesorado aprenda a trabajar mejor (a perfeccionar su práctica docente)" (LópezPastor, 2009, p. 35). Por otra parte, una de las características del modelo de EFyC es estar integrada en el proceso de E-A, con las ventajas que esto supone. Este tipo de evaluación supone "un proceso y una oportunidad de aprendizaje y debe hacer consciente al alumnado de su nivel competencial actual, pero también debe situar al docente sobre qué debe mejorar para enfrentarse a situaciones de aprendizaje futuras" (Manrique, Vallés y Gea, 2012, p. 88).

La EFyC tiene dos características complementarias: (a) el proceso de evaluación formativa, orientado a mejorar el aprendizaje de alumnado y profesorado, así como la mejora del proceso de enseñanza-aprendizaje llevado a cabo; y (b) el proceso de evaluación compartida, que supone la implicación del alumnado en los procesos de evaluación, a través de técnicas como la autoevaluación, la evaluación entre iguales y la evaluación compartida profesoralumno (López-Pastor, 2009).

En los procesos de EFyC hay elementos necesarios e indispensables, como el "feedback", que opera como un importante catalizador en la mejora continua del proceso de E-A (Carless, 2016).

Nicol y Macfarlane-Dick (2006) presentan un listado de siete principios de buenas prácticas de feedback, así como un análisis de la influencia de la evaluación formativa en los procesos de auto-regulación del aprendizaje. En trabajos similares también abordan la relación entre los procesos de evaluación formativa, auto-regulación del aprendizaje y utilización de tecnologías de aprendizaje (Nicol, 2009). Boud y Molloy (2013) analizan dos modelos de feedback: (a) donde el profesorado impulsa el feedback; (b) donde el alumnado adquiere un rol importante, solicitando su propio feedback. El estudio muestra que el segundo modelo es más efectivo, al generar oportunidades para el alumnado, alejándose de la creación de expectativas inalcanzables, y al entregar al alumnado la posibilidad de incidir en su aprendizaje. Por otra parte, Boud y Falchikov $(2006,2007)$ trabajan sobre el alineamiento de la evaluación formativa y su relación con el aprendizaje a largo plazo. Carless $(2007,2015)$ es un autor que ha generado numerosos trabajos sobre la propuesta denominada "Learning-oriented assessment" (LOA). Este autor señala que el LOA debe tener tres características principales: (1) las tareas de evaluación deben estimular el aprendizaje del alumnado; (2) implicar al alumnado en su propia evaluación y en la evaluación de otros compañeros; y (3) el feedback o retroalimentación ha de darse en el momento adecuado y debe ser útil para el aprendizaje actual y futuro.

El componente de evaluación compartida tiene cierta relación con lo que Aubert, Flecha, García, Flecha y Racionero (2009) denominan: "dialogo igualitario”, así como la mejora en la relación alumnado-profesorado que esto propicia. También pueden encontrarse otras referencias sobre este tipo de procesos, de promoción de la implicación del alumnado en la evaluación en trabajos de otros autores (Boud, 1995; Carless, 2007, 2015; Carless, Joughin y Mok, 2006; Gómez y Quesada, 2017).

Tras la declaración de Bolonia (1999), los sistemas por competencias son obligatorios en el Espacio Europeo de Educación Superior (EEES). En cambio en el ECES se adopta este tipo de currículo en 2005 por una universidad y, de manera paulatina, otras universidades 
chilenas lo han ido adoptando (Quezada, 2014). Por su parte, en la realidad Europea se exhiben una serie de experiencias evaluativas que sitúan a la EFyC como idónea para implementarse en modelos curriculares por competencias, ya que este tipo de evaluación coincide con el proceso y no centrarse solo en la etapa final. En este tipo de sistemas cobra real importancia el alumnado en la evaluación del proceso E-A, así también cobra importancia sus percepciones tanto a nivel de la valoración que hacen a los sistemas de evaluación de la que formaron parte, como en el grado de dominio competencial que alcanzan después de vivenciar sistemas de $\mathrm{EFyC}$, donde además podemos ver que el alumnado en algunos casos es más exigente que el propio profesorado, indicando una valoración de sus estados competenciales distinta, auto valorándose en un nivel más bajo vs. la valoración que le otorga el profesorado (Torelló, 2012), encontrándose incluso experiencias en donde el alumnado, además de auto percibirse como medianamente competente, baja esta valoración a medida que avanzan en su proceso formativo (Villamizar, Becerra y Delgado, 2016).

En el ECES no se encuentran experiencias evaluativas sistemáticas que den cuenta sobre que sistemas de evaluación resultan idóneos al aplicarse en asignaturas que se imparten en modelos por competencias, a pesar que la adopción de modelos por competencias lleva ya más de una década desde que la primera universidad chilena adoptara dicho modelo (Quezada, 2014).

El objetivo del estudio es conocer la percepción del alumnado sobre la aplicación de sistemas de Evaluación Formativa y Compartida en una universidad chilena, que se han llevado a cabo durante cuatro cursos seguidos en cuatro asignaturas diferentes.

\section{Método}

\subsection{Enfoque metodológico}

El enfoque metodológico utilizado en el estudio corresponde al enfoque cuantitativo, ya que como menciona Canales-Cerón (2006) "trabaja con unidades simples y equivalentes. Tanto en la muestra como en el instrumento, opera con números. Individuos-abstraídos de sus relaciones sociales, y abstraídos de su complejidad subjetiva” (p. 13), A su vez, la investigación fue de corte transversal, recolectando datos solo en la instancia final de cada asignatura, procediendo a analizar y describir las variables.

\subsection{Muestra y muestreo}

Las experiencias se desarrollaron con alumnado de Formación Inicial del Profesorado de Educación Física (FIPEF). La muestra correspondió al 100\% del alumnado que estaban cursando las asignaturas en las que se han utilizado sistemas de EFyC, en el momento de recolección de los datos. Concretamente se trata de 111 estudiantes, de los cuales 82 son varones y 29 mujeres. La edad media del alumnado es de 23.2 años, con una desviación típica $(d t$.) de 2.1. El tipo de muestreo fue no aleatorio y por conveniencia (López, 2004), quedando la muestra compuesta por alumnado de cuatro asignaturas de FIPEF de una universidad en la que se han implementado sistemas de EFyC (ver cuadro 1). 
Cuadro 1. Distribución de la muestra según año y semestre en el que se implemento la experiencia de $\mathrm{EFyC}$

\begin{tabular}{ccccc}
\hline AÑo & ASIGNATURA & $\begin{array}{c}\text { SEMESTRE EN } \\
\text { EL QUE SE } \\
\text { IMPARTE }\end{array}$ & $\begin{array}{c}\text { NúMERO DE } \\
\text { PARTICIPANTES }\end{array}$ & EDADES \\
\hline 2014 & Prácticum & $8^{\circ}$ Semestre & 24 alumnos y alumnas & $24.1 \pm 1.8$ \\
2015 & $\begin{array}{c}\text { Actividad Física y Salud para } \\
\text { Grupos Especiales } \\
\text { "Gasto Energético y } \\
\text { Antropometría" }\end{array}$ & $6^{\circ}$ Semestre & 19 alumnos y alumnas & $22 \pm 2.2$ \\
2016 & $\begin{array}{c}\text { "Gasto Energético y } \\
\text { Antropometría" }\end{array}$ & $6^{\circ}$ Semestre & 25 alumnos y alumnas & $23 \pm 1.9$ \\
& Semestre & 43 alumnos y alumnas & $23.2 \pm 2.1$ \\
\hline
\end{tabular}

Fuente: Elaboración propia.

\subsection{Técnica de recogida de información}

Se utilizo "el cuestionario" como técnica de investigación de campo, específicamente para determinar los efectos causados por el sistema de EFyC utilizado en cada asignatura. Se aplicó al alumnado el "Cuestionario de evaluación sobre la experiencia de buena práctica", al finalizar el correspondiente semestre académico. Se trata de un cuestionario previamente validado (Castejón-Oliva, Santos-Pastor y Palacios, 2015). El cuestionario utiliza una escala tipo likert de cinco grados, en donde $0=$ Nada; $1=$ Poco; $2=$ Algo; $3=$ Bastante; $4=$ Mucho, en respuesta a las afirmaciones contenidas en una serie de ítems. En el presente artículo se analizaron los ítems correspondientes a los factores de "Ventajas del sistema de evaluación" y los factores de "Posibles inconvenientes del sistema de evaluación", ambos factores estaban compuestos por una serie de ítems (16 y 14 respectivamente) que se refieren a una serie de características que pueden encontrarse en sistemas evaluativos, como se aprecia en el cuadro 2.

Cuadro 2. Factores del "Cuestionario de evaluación sobre la experiencia de buena práctica"

\begin{tabular}{|c|c|}
\hline $\begin{array}{c}\text { FACTOR VENTAJAS DEL SISTEMA DE } \\
\text { EVALUACIÓN }\end{array}$ & $\begin{array}{c}\text { FACTOR POSIBLES INCONVENIENTES DEL SISTEMA } \\
\text { DE EVALUACIÓN }\end{array}$ \\
\hline $\begin{array}{l}\text { 11. Señala si estás de acuerdo con las } \\
\text { siguientes afirmaciones relativas al } \\
\text { sistema de evaluación de la } \\
\text { asignatura: }\end{array}$ & $\begin{array}{l}\text { 12. Señala si estás de acuerdo con las siguientes } \\
\text { afirmaciones relativas al sistema de evaluación de la } \\
\text { asignatura: }\end{array}$ \\
\hline $\begin{array}{l}\text { 11.1. Ofrece alternativas a todos los } \\
\text { estudiantes }\end{array}$ & 12.1. Exige una asistencia obligatoria y activa \\
\hline $\begin{array}{l}\text { 11.2. Hay un contrato previo, } \\
\text { negociado y consensuado del } \\
\text { sistema de evaluación }\end{array}$ & $\begin{array}{l}\text { 12.2. Tiene una dinámica de trabajo poco conocida, } \\
\text { falta de hábito }\end{array}$ \\
\hline $\begin{array}{l}\text { 11.3. Esta centrado en el proceso, } \\
\text { importancia del trabajo diario }\end{array}$ & 12.3. Exige continuidad \\
\hline $\begin{array}{l}\text { 11.4. El estudiante realiza un } \\
\text { aprendizaje activo }\end{array}$ & 12.4. Hay que comprenderlo previamente \\
\hline $\begin{array}{l}\text { 11.5. Se plantea el trabajo en equipo } \\
\text { de forma colaborativa }\end{array}$ & 12.5. Exige un mayor esfuerzo \\
\hline $\begin{array}{l}\text { 11.6. El alumno está más motivado, } \\
\text { el proceso de aprendizaje es más } \\
\text { motivador }\end{array}$ & 12.6. Existe dificultad para trabajar en grupo \\
\hline 11.7. La calificación es más justa & 12.7. Se puede acumular mucho trabajo al final \\
\hline $\begin{array}{l}\text { 11.8. Mejora la tutela académica } \\
\text { (seguimiento y ayuda al alumno) }\end{array}$ & 12.8. Existe una desproporción trabajo/créditos \\
\hline
\end{tabular}




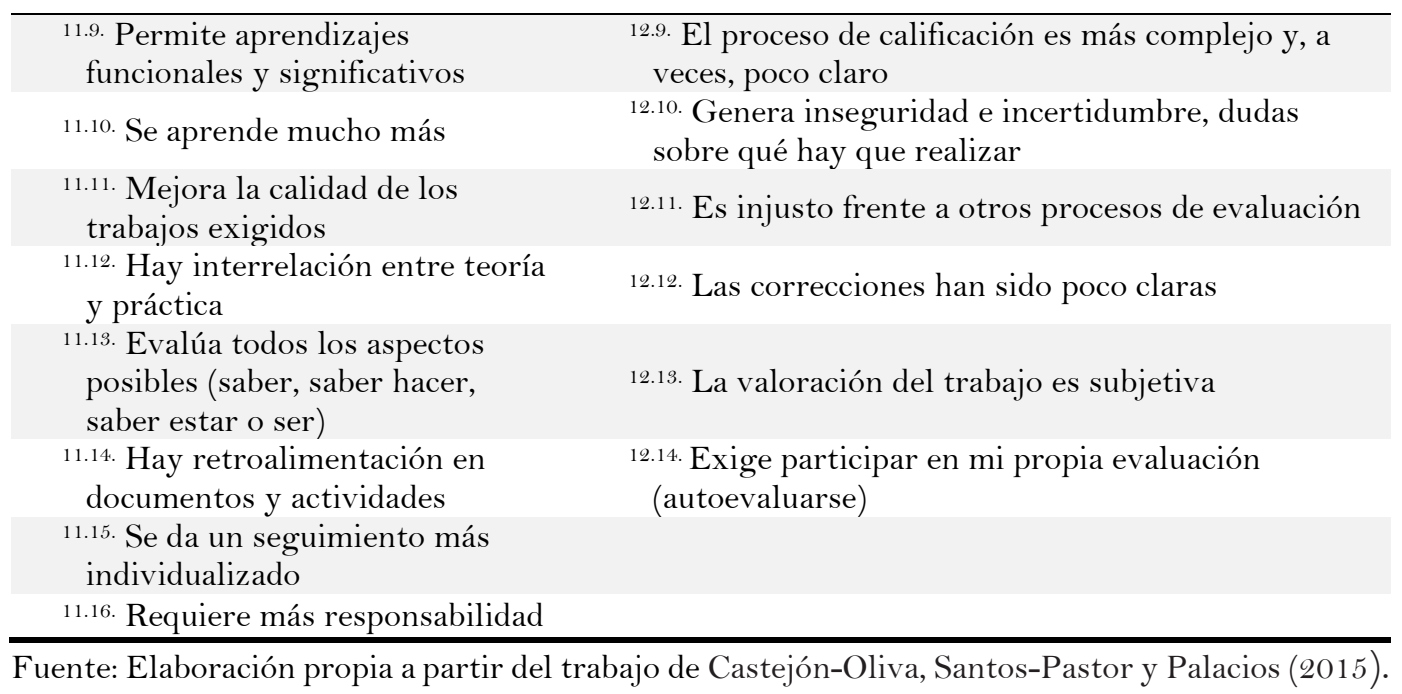

\subsection{Análisis de la información}

Para el análisis de los datos recogidos se aplicó un análisis de estadística descriptiva; específicamente se obtuvo la media aritmética $(M)$ y la desviación típica $(d t$.), tanto de cada uno de los cuatro cursos, como la media total entre todos los datos. Este análisis estadístico ha sido realizado con el software estadístico SPSS en su versión 18.0.

\section{Sistema de evaluación Formativa y Compartida utilizado}

Se utilizó un sistema de EFyC, dadas las múltiples experiencias en donde se han reportado mayormente ventajas tras la utilización de este tipo de evaluación (Castejón, LópezPastor, Julián y Zaragoza, 2011, López-Pastor, Pérez, Barba y Lorente, 2016; Martín, 2007), con experiencias que reportan estas ventajas en Chile (Gallardo-Fuentes y CarterThuillier, 2016), y recientes reportes que confirman su vigencia (Gallardo-Fuentes, López-Pastor y Carter-Tuhillier, 2018).

Los sistemas de EFyC utilizados se centraron en dos aspectos fundamentales: a) estar insertos en el proceso Enseñanza-Aprendizaje; b) Mantener un feedback constante e inmediato, para lo cual se utilizaron una serie de actividades de aprendizaje que se relacionaban con la estructura de evaluación con la que contaba la universidad en que se desarrolló el estudio (ver cuadro 3).

Tal como se aprecia en el cuadro 3, las actividades de aprendizaje están estrechamente relacionadas al feddback constante e inmediato, característica fundamental para que exista $\mathrm{EFyC}$. 
Cuadro 3. Actividades de aprendizaje, Evaluación Formativa y Feedback, instrumentos evaluativos utilizados y ponderación en la calificación final

\begin{tabular}{|c|c|c|c|c|}
\hline $\begin{array}{c}\text { ESTRUCTURA } \\
\text { DE } \\
\text { EVALUACIÓN } \\
\text { NORMADA POR } \\
\text { LA } \\
\text { UNIVERSIDAD }\end{array}$ & $\begin{array}{l}\text { ACTIVIDADES } \\
\text { DE } \\
\text { APRENDIZAJE }\end{array}$ & $\begin{array}{l}\text { EVALUACIÓN } \\
\text { FORMATIVA Y } \\
\text { FEEDBACK }\end{array}$ & $\begin{array}{c}\text { INSTRUMENTO } \\
\text { EVALUATIVOS } \\
\text { UTILIZADOS }\end{array}$ & $\begin{array}{l}\text { PESO EN LA } \\
\text { CALIFICACIÓN } \\
\text { FINAL }\end{array}$ \\
\hline $\begin{array}{l}\text { "Evaluaciones de } \\
\text { Proceso" }\end{array}$ & $\begin{array}{l}\text { Clases } \\
\text { expositivas } \\
\text { guiadas por el } \\
\text { Profesor de la } \\
\text { Cátedra } \\
\end{array}$ & $\begin{array}{l}\text { Feedback constante e } \\
\text { inmediato en talleres, } \\
\text { pruebas, tutorías, } \\
\text { interrogantes en la } \\
\text { clase. }\end{array}$ & $\begin{array}{l}\text { Escala de } \\
\text { puntos al } 60 \% \\
\text { exigencia }\end{array}$ & $40 \%$ \\
\hline $\begin{array}{l}\text { "Evaluación } \\
\text { integradora de } \\
\text { proceso" }\end{array}$ & $\begin{array}{l}\text { Clases } \\
\text { dirigidas por } \\
\text { el alumnado, } \\
\text { con } \\
\text { interacción } \\
\text { del profesor }\end{array}$ & $\begin{array}{l}\text { Feedback inmediato } \\
\text { una vez terminada la } \\
\text { exposición o actividad, } \\
\text { y retroalimentación } \\
\text { escrita a los } \\
\text { documentos entregados } \\
\text { por el alumnado. }\end{array}$ & Rubrica & $20 \%$ \\
\hline $\begin{array}{l}\text { "Evaluación } \\
\text { orientada al } \\
\text { desempeño } \\
\text { profesional" }\end{array}$ & $\begin{array}{l}\text { Diseño y } \\
\text { presentación } \\
\text { de Proyecto }\end{array}$ & $\begin{array}{l}\text { El alumnado diseña un } \\
\text { proyecto acorde a como } \\
\text { la asignatura en } \\
\text { cuestión impactara en } \\
\text { su futuro profesional, y } \\
\text { se entrega } \\
\text { retroalimentación en } \\
\text { horarios de tutoría. }\end{array}$ & Rubrica & $25 \%$ \\
\hline "Autoevaluación" & $\begin{array}{l}\text { Cierre con } \\
\text { reflexión } \\
\text { Alumnado- } \\
\text { Profesor }\end{array}$ & $\begin{array}{l}\text { Con interacción del } \\
\text { profesor el alumnado se } \\
\text { autoevalúa }\end{array}$ & $\begin{array}{l}\text { Pauta de } \\
\text { autoevaluación }\end{array}$ & $15 \%$ \\
\hline
\end{tabular}

Fuente: Elaboración propia.

\section{Resultados}

En el cuadro 4 se presentan los resultados correspondientes al bloque de afirmaciones del "Factor Ventajas del Sistema de Evaluación".

Como se aprecia en los resultados de la tabla 4, los ítems referentes a las "ventajas del sistema de evaluación" se presentan en los resultados generales para cada ítem con valoraciones altas (entre bastante y mucho), lo que indica la existencia de elementos que pueden considerarse como $\mathrm{EFyC}$ en los sistemas evaluativos que se utilizaron en las cuatro asignaturas. Las medias totales en todos los ítems oscilan entre el 2,6 y el 3.2; por tanto, dado que la escala es $0-4$, pueden considerarse puntuaciones altas y bastante similares, dado que hay poca disparidad entre unos ítems y otros. Se pueden observar dos bloques ligeramente diferenciados: (a) los que tienen puntuaciones entre 3,0 y 3,1 (ítems 1, 4, 9, 10, 12, 13, 14 y 16); y (b) los que tienen puntuaciones entre 2,6 y 2,9 (ítems $2,3,5,6,7,8$, 11 y 15$)$.

Dentro del bloque con las puntuaciones ligeramente más altas, destaca el ítem que afirma que: "Requiere más responsabilidad", que obtuvo la valoración general más elevada (3.1 sobre 4), lo que indica que a percepción del alumnado este tipo de evaluaciones les demanda una dedicación e implicación mayor que en otras asignaturas con modelos más 
tradicionales, donde no hay una exigencia de trabajo continua, sino que suele acumularse al final de la asignatura. También alcanzan valoraciones muy altas afirmaciones como: "ofrece alternativas a todos los estudiantes"; "el estudiante realiza un aprendizaje activo"; "permite aprendizajes funcionales y significativos"; "se aprende mucho mas"; "hay interrelación entre teoría y práctica"; "evalúa todos los aspectos posibles (saber, saber hacer, saber estar o ser)"; "hay retroalimentación en documentos y actividades". La alta valoración a estas afirmaciones, dejan ver el efecto positivo que la utilización de sistemas de $\mathrm{EFyC}$ parece tener en aspectos como un mayor y mejor aprendizaje, una mayor adaptación a las diferencias y circunstancias personales en los procesos de aprendizaje del alumnado y una mejor y más completa evaluación de los diferentes tipos de conocimientos y competencias.

Cuadro 4. Valoraciones al "Factor Ventajas del Sistema de Evaluación"

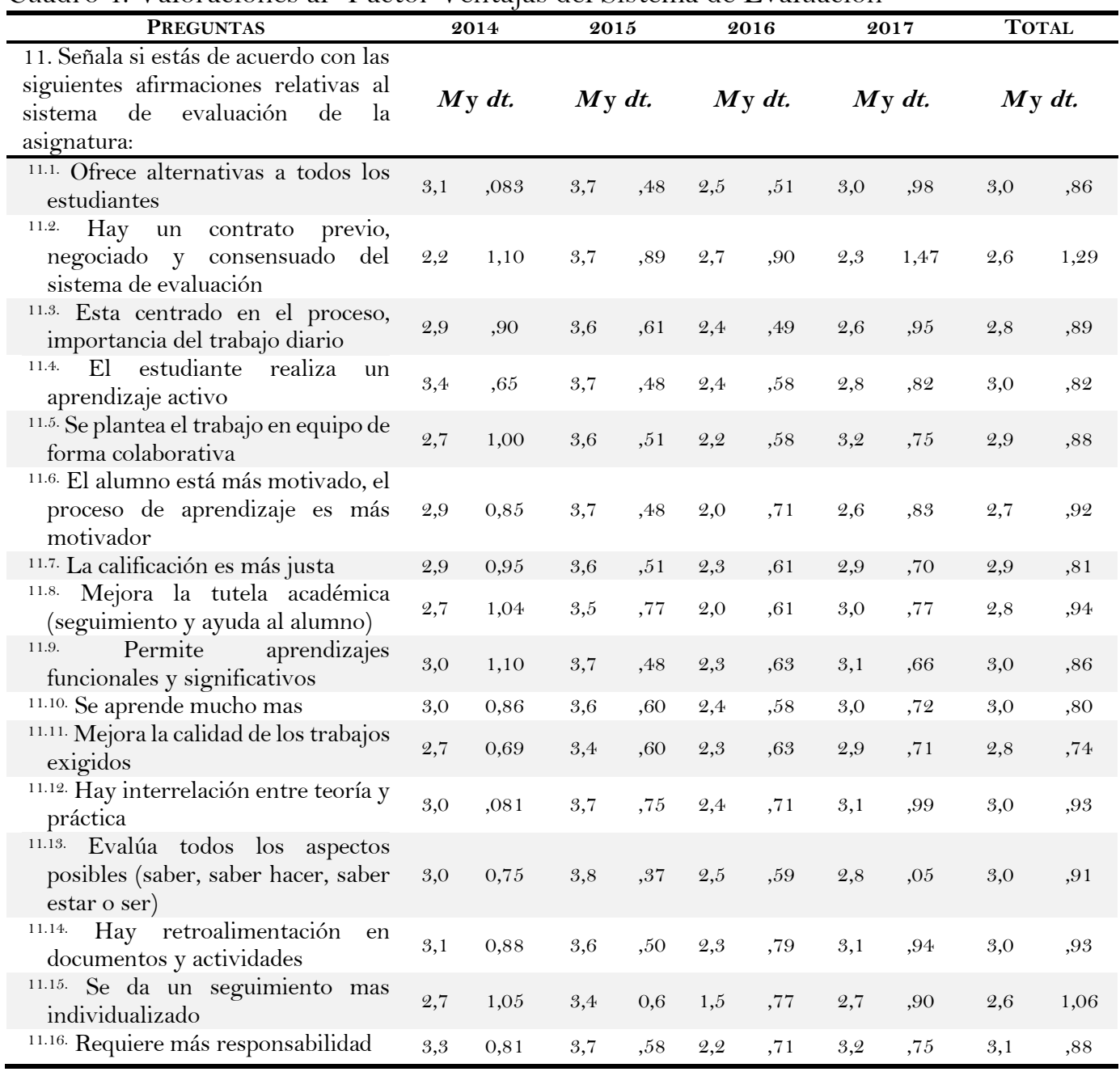

Fuente: Elaboración propia.

Dentro del bloque con las puntuaciones ligeramente más bajas, destacan los dos ítems que tienen una media inferior (2.6 sobre 4): "Hay un contrato previo, negociado y consensuado del sistema de evaluación" y "Se da un seguimiento más individualizado". Esta valoración está entre "algo" y "bastante", por lo que, a pesar de ser los valores más bajos, indica que se cumplen en un alto grado las dos cosas, aunque se perciba como ligeramente menor que 
otras características de los sistemas de de $\mathrm{EFyC}$ vividos. El resto de ítems se mueven en valores muy cercanos entre sí (2,7-2,9), y muestran valoraciones altas en aspectos como: la importancia del trabajo diario para lograr procesos de aprendizaje continuo, la importancia del trabajo colaborativo para un mejor aprendizaje y una mayor motivación, el mejor seguimiento del alumnado y la mayor justicia en la calificación final.

Por otra parte, si comparamos los resultados de los diferentes cursos académicos, podemos comprobar cierta variedad de resultados por ítems y cursos, aunque pueden observarse ciertas generalidades. Por ejemplo, que el curso 2016 tiene las medias globales más bajas de los cuatro en muchos de los ítems (no en todos); mientras que el curso 2015 es el que tiene las medias más altas, en algunos ítems muy altas (entre 3,4 y 3,8). Creemos que estas diferencias pueden estar influenciadas principalmente la naturaleza de la asignatura, ya que la asignatura del año 2015 (“Actividad Física y Salud para Grupos Especiales”) es teórico-práctica, con una alta carga de elementos didácticos, que buscan preparar al alumnado para el trabajo en aulas con presencia de población con necesidades educativas especiales; mientras que, la asignatura del año 2016 ("Gasto Energético y Antropometría") es de corte teórico y correspondiente a la línea de la especialidad, donde la falta de instancias practicas pudo ser un aspecto didáctico que influyo en la valoración del alumnado. Finalmente no creemos que el curso en el que se encuentran las asignaturas influya, considerando que tanto la asignatura del 2015 como la del 2016 se imparten el mismo semestre $\left(6^{\circ}\right.$ semestre).

En el cuadro 5 se presentan los resultados del bloque de afirmaciones que responden al "Factor Posibles Inconvenientes del Sistema de Evaluación".

Tal y como se aprecia en el cuadro 5, los ítems que responden al factor de "Posibles Inconvenientes del Sistema de Evaluación” se presentan en resultados generales con valoraciones muy variadas, pudiéndose observar que las medias totales para cada ítem oscila entre poco y bastante $(1,3-3,0)$. Se puede apreciar la presencia de tres bloques de ítems según la valoración recibida. En un primer bloque están las afirmaciones que obtuvieran valoraciones altas (entre algo y bastante) donde encontramos: "Exige una asistencia obligatoria y activa" (2.9 sobre 4); "Exige continuidad" (2.9 sobre 4); "Hay que comprenderlo previamente" (2,7 sobre 4); "Exige un mayor esfuerzo" (2,8 sobre 4); y "Exige participar en mi propia evaluación (autoevaluarse)" (3,0 sobre 4), por lo que se puede apreciar que, a juicio del alumnado, los sistemas de $\mathrm{EFyC}$ requieren de continuidad, comprensión previa, mayor esfuerzo, exigen participar de la evaluación, pudiendo acumularse mucho trabajo al final del proceso. Todas estas afirmaciones confirman la presencia de elementos que pueden considerarse como de $\mathrm{EFyC}$, claramente diferentes a las características de los sistemas tradicionales, en donde suele acumularse y trasladarse la evaluación al final del proceso de E-A.

En un segundo bloque se encuentra un grupo de afirmaciones que obtuvieron valoraciones medias, lo que indica que a juicio del alumnado ocurrió entre poco y algo. Dentro de las afirmaciones que obtuvieran estas valoraciones medias podemos encontrar: "Tiene una dinámica de trabajo poco conocida, falta de hábito" (2,2 sobre 4); "Existe dificultad para trabajar en grupo" (2,0 sobre 4); "Se puede acumular mucho trabajo al final” ( 2,1 sobre 4$)$. Estos posibles inconvenientes suelen apreciarse en alumnado que vivencia por primera vez sistemas de EFyC. Los otros ítems de este grupo que presentara valoraciones medias son levemente más bajos, acá nos encontramos con los siguientes: "Existe una 
desproporción trabajo/créditos" (1,9 sobre 4); "El proceso de calificación es más complejo y, a veces, poco claro" (1,8 sobre 4); "Genera inseguridad e incertidumbre, dudas sobre qué hay que realizar" (1,9 sobre 4); "La valoración del trabajo es subjetiva” (1.8 sobre 4). En estos ítems se observa que, a juicio del alumnado, en sólo en pocas o algunas ocasiones el sistema de $\mathrm{EFyC}$ les pareció complejo e injusto. También se aprecia la presencia de elementos de evaluación compartida, ya que el alumnado manifiesta que solo en pocas o algunas veces la valoración de sus trabajos fue subjetiva.

Cuadro 5. Valoraciones al Factor Posibles Inconvenientes del Sistema de Evaluación

\begin{tabular}{|c|c|c|c|c|c|c|c|c|c|c|}
\hline Preguntas & \multicolumn{2}{|c|}{2014} & \multicolumn{2}{|c|}{2015} & \multicolumn{2}{|c|}{2016} & \multicolumn{2}{|c|}{2017} & \multicolumn{2}{|c|}{ TotaL } \\
\hline $\begin{array}{l}\text { 12. Señala si estás de acuerdo con las } \\
\text { siguientes afirmaciones relativas al } \\
\text { sistema de evaluación de la asignatura: }\end{array}$ & \multicolumn{2}{|c|}{ M y dt. } & \multicolumn{2}{|c|}{ M y dt. } & \multicolumn{2}{|c|}{ M y dt. } & \multicolumn{2}{|c|}{ M y dt. } & \multicolumn{2}{|c|}{ M y dt. } \\
\hline $\begin{array}{l}\text { 12.1. Exige una asistencia obligatoria y } \\
\text { activa }\end{array}$ & 3,0 & 0,88 & 3.7 & 0,48 & 2,4 & 0,58 & 2,8 & 1,18 & 3,9 & 0,99 \\
\hline $\begin{array}{l}\text { 12.2. Tiene una dinámica de trabajo poco } \\
\text { conocida, falta de hábito }\end{array}$ & 2,1 & 1,23 & 2.9 & 1,03 & 1,6 & 0,82 & 2,2 & 1,10 & 2,2 & 1,13 \\
\hline 12.3. Exige continuidad & 3,0 & 0,83 & 3.7 & 0,58 & 2,3 & 0,74 & 2,7 & 1,08 & 2,9 & 0,98 \\
\hline $\begin{array}{l}\text { 12.4. Hay que comprenderlo } \\
\text { previamente }\end{array}$ & 2,8 & 0,92 & 3.4 & 0,77 & 2,0 & 0,61 & 2,7 & 0,88 & 2,7 & 0,93 \\
\hline 12.5. Exige un mayor esfuerzo & 2,6 & 0,97 & 3.5 & 0,61 & 2,0 & 0,79 & 3,0 & 0,87 & 2,8 & 0,96 \\
\hline $\begin{array}{l}\text { 12.6. Existe dificultad para trabajar en } \\
\text { grupo }\end{array}$ & 1,5 & 1,02 & 2.6 & 1,22 & 1,0 & 1,02 & 2,7 & 1,13 & 2,0 & 1,29 \\
\hline $\begin{array}{l}\text { 12.7. Se puede acumular mucho trabajo } \\
\text { al final }\end{array}$ & 2,1 & 0,80 & 2.6 & 1,30 & 1,2 & 0,93 & 2,4 & 1,16 & 2,1 & 1,16 \\
\hline $\begin{array}{l}\text { 12.8. Existe una desproporción } \\
\text { trabajo/créditos }\end{array}$ & 1,6 & 1,10 & 2.4 & 1,50 & 1,2 & 1,20 & 2,2 & 0,99 & 1,9 & 1,23 \\
\hline $\begin{array}{l}\text { 12.9. El proceso de calificación es más } \\
\text { complejo y, a veces, poco claro }\end{array}$ & 1,5 & 0,83 & 2.4 & 1,50 & 0,8 & 0,90 & 2,2 & 1,27 & 1,8 & 1,29 \\
\hline $\begin{array}{l}\text { 12.10. Genera inseguridad e } \\
\text { incertidumbre, dudas sobre qué hay } \\
\text { que realizar }\end{array}$ & 1,4 & 1,02 & 2.2 & 1,27 & 1,0 & 0,82 & 2,1 & 1,04 & 1,7 & 1,13 \\
\hline $\begin{array}{l}\text { 12.11. Es injusto frente a otros procesos } \\
\text { de evaluación }\end{array}$ & 1,2 & 1,17 & 1.8 & 1,51 & 0,7 & 0,98 & 1,6 & 1,26 & 1,3 & 1,27 \\
\hline $\begin{array}{l}\text { 12.12. Las correcciones han sido poco } \\
\text { claras }\end{array}$ & 1,3 & 1,03 & 2.0 & 1,41 & 0,6 & 0,87 & 1,7 & 1,24 & 1,4 & 1,25 \\
\hline $\begin{array}{l}\text { 12.13. La valoración del trabajo es } \\
\text { subjetiva }\end{array}$ & 1,6 & 1,18 & 2.5 & 1,50 & 1,1 & 0,93 & 1,9 & 1,32 & 1,8 & 1,31 \\
\hline $\begin{array}{l}\text { 12.14. Exige participar en mi propia } \\
\text { evaluación (autoevaluarse) }\end{array}$ & 3,3 & 0,62 & 2.9 & 1,22 & 2,4 & 0,65 & 3,2 & 0,91 & 3,0 & 0,92 \\
\hline
\end{tabular}

Fuente: Elaboración propia.

En el tercer bloque se aprecian las afirmaciones que obtuvieran valoraciones bajas, por ejemplo: "Es injusto frente a otros procesos de evaluación" (1,3 sobre 4) y "Las correcciones han sido poco claras" (1,4 sobre 4). Estos ítems presentan las valoraciones más bajas en las medias totales, por lo que podrían considerarse como datos positivos, ya que están indicando que en muy pocas ocasiones consideran que el sistema de evaluación es injusto o que las correcciones han sido poco claras. También se puede apreciar que en este último factor (Posibles Inconvenientes del Sistema de Evaluación) las valoraciones más bajas se encontraron con el alumnado que cursó la asignatura "Gasto Energético y Antropometría" en el año 2016, con una valoración entre nada y poco para dos ítems: "Es injusto frente a otros procesos de evaluación" y "Las correcciones han sido poco claras" ( 0,7 y 0,6 respectivamente), lo que indica que, a juicio del alumnado, esto no ocurrió durante la evaluación de la asignatura. 
Por otra parte, si comparamos los resultados de los diferentes cursos académicos, vuelven a observarse resultados muy similares a los ya explicados en la tabla de ventajas. Como puede comprobarse, de nuevo se comprueba cierta variedad de resultados por ítems y por cursos. A pesar de ello, las generalidades observadas son muy similares a las de la tabla anterior, en el sentido de que vuelve a ser el curso 2016 el que tiene las medias globales más bajas de los cuatro en muchos de los ítems (no en todos); mientras que es el curso 2015 el que tiene las medias más altas en la mayoría de los ítems. Por tanto, otra posible interpretación es que se trata de generaciones que tienen tendencia a puntuar más bajo o más alto, según el año.

\section{Discusión}

En los resultados que se presentan como ventajas del sistema de evaluación, a pesar de la presencia de dos bloques en función de las medias generales, dejan ver una alta valoración para todos los ítems de este factor, estos resultados son similares a múltiples estudios previos que han incorporado sistemas de EFyC en Chile y en España, tanto en el FIP como en todos los niveles formativos (Atienza, Valencia-Peris, Marts-García, LópezPastor y Devís-Devís, 2016; Gallardo-Fuentes, López-Pastor y Carter-Tuhillier, 2018; Hortigüela-Alcala, Pérez-Pueyo y Abella, 2015a; López-Pastor y Pérez-Pueyo, 2017; Luis-Pascual y Muros, 2018 ).

Se puede apreciar que en el factor "Ventajas del sistema de evaluación" la media general más elevada corresponde a la afirmación "Requiere más responsabilidad". Esto también se puede apreciar en otras investigaciones que utilizaran sistemas de $\mathrm{EFyC}$, y existen reportes consistentes al respecto, como la revisión que hicieran Hamodi, López y LópezPastor (2014), quienes analizaron en los últimos diez años de la implementación de EFyC en el ámbito universitario por la red de $\mathrm{EFyC}$, pudiéndose detectar que el alumnado manifiesta una percepción de mayor carga de trabajo, al igual que el profesorado. Pero los datos muestran que con la incorporación de EFyC no se sobreasan los créditos ECTS de las asignaturas, y aunque la responsabilidad aumenta en el alumnado, esto se debe a que la carga del trabajo se distribuye en todo el proceso de E-A y no solo en la etapa final.

Referente al ítem de ventajas del sistema de evaluación hay dos afirmaciones ("Hay un contrato previo, negociado y consensuado del sistema de evaluación” y "Se da un seguimiento más individualizado") que a pesar de obtener valoraciones que se ubican entre "algo y bastante", son las que obtuvieron medias generales más bajas, por lo que a juicio del alumnado el componente "compartido" está presente, pero es el que menos destaca en las experiencias de evaluación en las que participó. Esto requiere de una preocupación especial para futuras experiencias si se considera que la participación del alumnado es considerada por autores como Benito, Villaverde, Hortigüela y Abella-García (2016) como una característica de vital importancia en la evaluación de los procesos de E-A que se instalan en el nivel universitario, sobre todo si se considera que ayuda a poner acento en el carácter formativo de la evaluación (Gil y Padilla, 2009). Así, también investigaciones manifiestan que la implicación del alumnado ayuda a generar mayor vinculación del mismo alumnado con su propio proceso de E-A (Angelini, 2016; Atienza, Valencia-Peris, Martos-García, López-Pastor y Devís-Devís, 2016; Zaragoza, Luis-Pascual y Manrique, 2009), aunque se deja ver que a pesar de estas consideraciones se han encontrado evidencias de poca participación del alumnado posteriormente en los procesos de 
calificación (Cañadas, Santos-Pastor y Castejón, 2017; Lukas, Santiago, Lizasoain y Etxeberria, 2017; Martínez-Muñoz, Santos-Pastor y Castejón, 2017).

Respecto a los resultados del factor correspondiente a los "posibles inconvenientes del sistema de evaluación”, se comprueba que hay tres bloques de valoraciones:

a) Un bloque con altas valoraciones: En este bloque encontramos las afirmaciones "Exige una asistencia obligatoria y activa"; "Exige continuidad"; "Hay que comprenderlo previamente"; "Exige un mayor esfuerzo" y "Exige participar en mi propia evaluación (autoevaluarse)”. Se puede ver que este tipo de dificultades suele presentarse tras la aplicación de sistemas de EFyC (Fraile y Cornejo, 2013; Romero-Martín, Castejón-Oliva y López-Pastor, 2015; Hortigüela-Alcala, PérezPueyo y Abella-García; 2015a), así como en realidades que inician la implementación de EFyC por la novedad que esta supone (López-Pastor, et al., 2006). Existen experiencias en que el alumnado indica sentirse satisfecho con el sistema de evaluación que ha experimentado, a pesar de manifestar la presencia de estas dificultades (Gallardo-Fuentes y Carter-Thuillier, 2016); parece que, aunque el alumnado usualmente percibe este tipo de afirmaciones tras vivenciar sistemas de $\mathrm{EFyC}$ como posibles inconvenientes, son más bien características que todo sistema de EFyC posee (López-Pastor, 2009).

b) Un bloque con valoraciones medias donde se encuentran las afirmaciones: "Tiene una dinámica de trabajo poco conocida, falta de hábito"; "Existe dificultad para trabajar en grupo", lo que se comporta en línea con un estudio que encontró bajas valoraciones a estos ítems después de analizar las valoraciones que 3.013 alumnos(as) le dieran a los sistemas de $\mathrm{EFyC}$ de los que fueron parte (RomeroMartín, Castejón-Oliva, López-Pastor, 2015). Para las afirmaciones; "Se puede acumular mucho trabajo al final" y "Existe una desproporción trabajo/créditos", las valoraciones están en línea con una serie de investigaciones que, a pesar de que el alumnado encuentra una desproporción trabajo/créditos, suele ser solo una percepción del alumnado, quizás por la costumbre de someterse a sistemas de evaluación tradicionales que concentran toda la carga del trabajo evaluativo al final del semestre, pero sin embargo, al analizar la carga de trabajo que los sistemas de EFyC suponen, esta se encuentra dentro de lo considerado en la carga de trabajo que estipulan los ECTS (Atienza, Valencia-Peris, Martos-García, López-Pastor y Devís-Devís, 2016; López-Pastor et al, 2013; Manrique, Vallés y Gea, 2012; Romero et al, 2015). Y para las valoraciones; "El proceso de calificación es más complejo y, a veces, poco claro"; "Genera inseguridad e incertidumbre, dudas sobre qué hay que realizar"; "La valoración del trabajo es subjetiva", se pueden encontrar investigaciones que reportan resultados similares: en el estudio de Romero-Martín, Castejón-Oliva y López-Pastor (2015) se puede apreciar como en una muestra muy numerosa (3.013 estudiantes universitarios) se encontraron resultados que desechan ciertos prejuicios respecto a la $\mathrm{EFyC}$, específicamente en torno a la complejidad y las sensaciones de inseguridad e incertidumbre que generan; incluso en este estudio el alumnado manifiesta que la valoración de sus trabajos no fue tan subjetiva y destacan la justicia del proceso y la claridad de las correcciones. 
c) Un tercer bloque con bajas valoraciones: en este bloque se encontraron dos afirmaciones que recibieran valoraciones indicando que, a juicio del alumnado, ocurren poco. Acá encontramos la afirmación "Es injusto frente a otros procesos de evaluación" y "Las correcciones han sido poco claras", el alumnado entrego una baja valoración a estas afirmaciones, al igual que ocurriera en el estudio de Hortigüela-Alcalá, Perez-Pueyo y Abella-García (2015a) donde tras analizar las valoraciones de 3.618 alumnos de 17 universidades y las correspondientes 52 asignaturas que utilizaran sistemas de EFyC, se encontraron con un $67.2 \%$ del alumnado que indicara mucho en una escala de: Nada (1) Poco (2) Algo (3) Bastante (4) Mucho (5), al momento de valorar la presencia de un elemento fundamental en todo sistema de EFyC, como lo es "la retroalimentación".

\section{Conclusiones}

A modo de conclusión se ha cumplido con el objetivo de investigación previamente establecido, que hace referencia a "conocer la percepción del alumnado sobre la aplicación de sistemas de Evaluación Formativa y Compartida en una universidad chilena, que se han llevado a cabo durante cuatro cursos seguidos en cuatro asignaturas diferentes". Los datos muestran que el alumnado de FIPEF suele dar valoraciones más altas a las afirmaciones que hacen referencia a las ventajas del uso de sistemas de EFyC durante su formación inicial. A juicio del alumnado participante el ítem que recibe la valoración más elevada de forma general hace referencia a la mayor responsabilidad que requiere trabaja con estos sistemas de evaluación, entendiéndose esta elevada responsabilidad como un factor positivo para su proceso formativo. Aunque con valoraciones casi igual de altas pueden encontrarse los ítems que hacen referencia a aspectos como ofrecer alternativas a todos los estudiantes y que ofrece un feedback continuo, lo que ayuda a realizar mejores procesos de aprendizaje (más aprendizaje, más activo, funcional y significativo) y que realizan una evaluación más completa, que evalúa los diferentes tipos de aprendizajes y competencias. Con valoraciones ligeramente más bajas (pero altas en términos generales), pueden encontrarse los ítems que hacen referencia a aspectos como la existencia de procesos de negociación curricular sobre el sistema de evaluación a utilizar, la importancia del trabajo diario y colaborativo para lograr procesos de aprendizaje mejores, más continuos y con una mayor motivación, el mejor seguimiento del alumnado y la mayor justicia en la calificación final.

Por su parte las afirmaciones del factor "Posibles Inconvenientes del Sistema de Evaluación”, que recibieran valoraciones elevadas, lo hacen en aspectos como la asistencia obligatoria, la continuidad, comprensión previa, el esfuerzo que demanda y otras exigencias que son más bien consideradas características de todo sistema de EFyC. Además, cómo ya se ha visto en otros estudios, esta percepción de mayor carga de trabajo se ve recompensada con un mayor aprendizaje.

El estudio puede ser de interés para el profesorado que trabaja en FIP y FIPEF de titulaciones que funcionen bajo un modelo de aprendizaje por competencias y que esté interesado en implementar sistemas de $\mathrm{EFyC}$ en sus aulas, como vía para potenciar mejores procesos de aprendizaje en su alumnado. También puede ser de interés para los investigadores especializados en este tipo de temáticas, dado que ofrece datos empíricos de universidades donde existen muy pocas referencias publicadas sobre la temática. 
Como prospectiva, parece interesante analizar los efectos de la EFyC comparando asignaturas que no utilizan $\mathrm{EFyC}$ con asignaturas donde sí se utilizan estos sistemas evaluativos. Otra posible vía de trabajo e investigación puede ser trasferir este tipo de experiencias sobre la utilización de sistemas de EFyC en asignaturas que se impartan en titulaciones distintas a las de FIPEF.

\section{Referencias}

Angelini, L. (2016). Estudio sobre la evaluación formativa y compartida en la formación docente en inglés. Actualidades Investigativas en Educación, 16(1), 1-21.

Atienza, R., Valencia-Peris, A., Martos-García, D., López-Pastor, V. y Devís-Devís, J. (2016). La percepción del alumnado universitario de educación física sobre la evaluación formativa: ventajas, dificultades y satisfacción. Movimento, 22(4), 1033-1048.

Aubert, A., Flecha, A., García, C., Flecha, R. y Racionero, S. (2009). Aprendizaje dialógico en la Sociedad de la Información. Barcelona: Hipatia.

Ayala, R., Messing, H., Labbé, C. y Obando, N. (2010). Congruencia entre el diseño curricular y la evaluación de los aprendizajes esperados en cátedras impartidas en una universidad chilena. Estudios pedagógicos, 36(1), 53-67. https://doi.org/10.4067/So718-07052010000100002

Benito, V., Villaverde, V., Hortigüela, D. y Abella-García, V. (2016). Evaluación entre iguales: Una experiencia de evaluación compartida en Educación Superior. EDUCADI, 1(1), 9-24.

Boud, D. (1995). Enhancing Learning through Self-assessment. Nueva York, NY: Routledge.

Boud, D. y Falchikov, N. (2006). Aligning assessment with long-term learning. Assessment $\mathcal{E}^{\circ}$ Evaluation in Higher Education, 25(3), 279-291.

Boud, D. y Falchikov, N. (2007).Rethinking Assessment in Higher Education.Learning for the long term. Oxon: Routledge.

Boud, D. y Molloy, E. (2013). Rethinking models of feedback for learning: The challenge of design. Assessment and Evaluation in Higher Education, 38(6), 698-712. https://doi.org/10.1080/02602938.2012.691462

Cabalín-Silva, D. y Navarro-Hernández, N. (2008). Conceptualización de los Estudiantes sobre el Buen Profesor Universitario en las Carreras de la Salud de la Universidad de La FronteraChile. International Journal of Morphology, 26. 887-892. https://doi.org/10.4067/S0717-95022008000400017

Canales-Cerón, M. (2006). Metodologías de la investigación social. Santiago: LOM ediciones.

Cano, M. (2008). La evaluación por competencias en la educación superior. Profesorado. Revista de Currículum y Formación de Profesorado, 12(3), 1-16.

Cañadas, L., Santos-Pastor, M. y Castejón, F. (2017). Diferencias en las formas de calificación empleadas por el profesorado universitario. Infancia. Educación y Aprendizaje, 3(2), 600-605. https://doi.org/10.22370/ieya.2017.3.2.787

Carless, D. (2007). Learning-oriented assessment: conceptual bases and practical implications. Innovations in Education and Teaching International, 44(1), 57-66.

Carless, D. (2015). Exploring learning-oriented assessment processes. Higher Education, 69(6), 963-976. 
Carless, D. (2016). Feedback as dialogue. Encyclopedia of educational philosophy and theory, 1-6. University of Hong Kong, Hong Kong. http://doi.org/10.1007/978-981-287-532-7_389-1

Carless, D., Joughin, G. y Mok, M. (2006). Learning-oriented assessment: principles and practice. Assessment $\Xi^{2}$ Evaluation in Higher Education, 31 (4), 395-398. https://doi.org/10.1080/02602930600679043.

Castejón-Oliva, F., López-Pastor, V., Julián, J. y Zaragoza, J. (2011). Evaluación formativa y rendimiento académico en la formación inicial del profesorado de educación física. International Journal of Medicine and Science of Physical Activity and Sport, 11(42), 238- 346.

Castejón-Oliva, F., Santos-Pastor, M. y Palacios, A. (2015). Cuestionario sobre metodología y evaluación en formación inicial en educación física. Revista Internacional de Medicina y Ciencias de la Actividad Física y del Deporte, 15(58), 245-267.

Declaration Bolonia. (1999). "The European Higher Education Area”, joint declaration of the European Ministers of Education, convened in Bologna, 19. Recuperado de https://www.eurashe.eu/library/bologna_1999_bologna-declaration-pdf/

Fraile, A. y Cornejo, P. (2013). La evaluación formativa en la enseñanza universitaria: una experiencia de innovación educativa con estudiantes de Educación Física. Revista de Evaluación Educativa, $1(2), 22-43$.

Gallardo-Fuentes, F. y Carter-Thuillier, B. (2016). La evaluación formativa y compartida durante el prácticum en la formación inicial del profesorado: Análisis de un caso en Chile. Retos. Nuevas tendencias en Educación Física Deportes y Recreación, 29, 258-263.

Gallardo-Fuentes, F., López-Pastor, V. y Carter-Tuhillier, B. (2018). Efectos de la Aplicación de un Sistema de Evaluación Formativa en la Autopercepción de Competencias Adquiridas en Formación Inicial del Profesorado. Estudios Pedagógicos, 44(2), 55-77. https://doi.org/10.4067/S0718-07052018000200055

Gil, J. y Padilla, M. (2009). La participación del alumnado universitario en la evaluación del aprendizaje. Educación XX1, 12, 43-65. https://doi.org/10.5944/educxx 1.1.12.287

Gómez, M. A. y Quesada, V. (2017). Coevaluación o Evaluación Compartida en el Contexto Universitario: La Percepción del Alumnado de Primer Curso. Revista Iberoamericana de Evaluación Educativa, 1O(2), 9-30. https://doi.org/10.15366/riee2017.10.2.001

Hamodi Galán, C., López Pastor, A. T., López-Pastor, V. M. (2014). Red de evaluación formativa y compartida en docencia universitaria: creación, consolidación y líneas de trabajo. Revista de evaluación educativa, 3(1).

Hernandez-Elizondo, J. y Salicetti-Fonseca, A. (2018). La Evaluación Formativa en el Proceso Enseñanza-aprendizaje en Estudiantes de Actividad Deportiva de la Universidad de Costa Rica. Estudios Pedagógicos, 44(2), 297-310. https://doi.org/10.4067/S0718-07052018000200297

Hortigüela-Alcalá, D., Pérez-Pueyo, Á. y Abella-García, V. (2015a). ¿De qué manera se implica el alumnado en el aprendizaje? Análisis de su percepción en procesos de evaluación formativa. Revista de Investigación en Educación, 13(1), 88-104.

Hortigüela-Alcalá, D., Perez-Pueyo, Á. y Abella-García, V. (2015b). Perspectiva del alumnado sobre la evaluación tradicional y la evaluación formativa: contraste de grupos en las mismas asignaturas. REICE: Revista Iberoamericana sobre Calidad, Eficacia y Cambio en Educación, 13(1), 35-48.

López, P. (2004). Población muestra y muestreo. Punto Cero, 9(8), 69-74. 
López-Pastor, V. y Pérez-Pueyo, Á. (2017). Evaluación formativa y compartida en educación: Experiencias de éxito en todas las etapas educativas. Recuperado de http://buleria.unileon.es/handle/10612/5999

López-Pastor, V., Monjas-Aguado, R., Gómez-García, E., López-Pastor, J., Martin-Pinela, J., González-Badiola, J., Barba-Martin, R., Aguilar-Baeza, R, González-Pascual, M., HerasBernardino, C., Martín, M., Manrique-Arribas J., Subtil-Marugán, P. y Marugán-García, L. (2006). La Evaluación en Educación Física. Revisión de los modelos tradicionales y planteamiento de una alternativa: La evaluación formativa y compartida. Retos. Nuevas Tendencias en Educación Física, Deporte y Recreación, 10, 31-41.

López-Pastor, V., Pérez, Á., Barba, J. y Lorente, E. (2016). Percepción del alumnado sobre la utilización de una escala graduada para la autoevaluación y coevaluación de trabajos escritos en la formación inicial del profesorado de educación física (FIPEF). Cultura, Ciencia y Deporte, $11(31), 37-50$.

López-Pastor, V., Pintor, P., Muros, B. y Webb, G. (2013): Formative assessment strategies and their effect on student performance and on student and tutor workload: the results of research projects undertaken in preparation for greater convergence of universities in Spain within the European Higher Education Area (EHEA). Journal of Further and Higher Education, 37(2), 163-180. https://doi.org/10.1080/0309877X.2011.644780

López-Pastor, V.M., Castejón, J., Sicilia-Camacho, A., Navarro, V. y Webb, G. (2011). The process of creating a cross-university network for formative and shared assessment in higher education in Spain and its potential applications. Innovations in Education and Teaching Internacional, 48(1), 79-90. https://doi.org/10.1080/14703297.2010.543768

Luis-Pascual, J. y Muros, B. (2018). La autocalificación como instrumento de aprendizaje en una asignatura universitaria inversa. Estudios Pedagógicos, 44(2), 79-91.

https://doi.org/10.4067/S0718-07052018000200079

Lukas, J., Santiago, K., Lizasoain, L. y Etxeberria, J. (2017). Percepciones del alumnado universitario sobre la evaluación. Bordón. Revista de Pedagogía, 69(1), 103-122. https://doi.org/10.13042/Bordon.2016.43843

Manrique, J., Vallés, C. y Gea, J. (2012). Resultados generales de la puesta en práctica de 29 casos sobre el desarrollo de sistemas de evaluación formativa en docencia universitaria. Psychology, Society \& Education, 4(1), 87-102.

Martín, P. (2007). Evaluación formativa y su repercusión en el clima del aula. Revista de Investigación Educativa, 25(2), 389-402.

Martínez-Muñoz, L., Santos-Pastor, M. y Castejón, F. (2017). Percepciones de alumnado y profesorado en Educación Superior sobre la evaluación en formación inicial en educación física. Retos. Nuevas Tendencias en Educación Física, Deporte y Recreación, 32, 76-81.

Nicol, D. (2009). Assessment for learner self-regulation: Enhancing achievement in the first year using learning technologies. Assessment E Evaluation in Higher Education, 34(3), 335-352.

Nicol, D. J. y Macfarlane-Dick, D. (2006). Formative assessment and self-regulated learning: A model and seven principles of good feedback practice. Studies in higher education, 31(2), 199218.

Quezada, I. (2014). El modelo de educación por competencias y su impacto en la planificación estratégica de la Universidad de Talca (Chile). Revista Universitaria Ruta, 16(1), 7-18.

Romero-Martín, M., Castejón-Oliva, F. y López-Pastor, V. (2015). Divergencias del alumnado y del profesorado universitario sobre las dificultades para aplicar la evaluación formativa. Revista 
Electrónica de Investigación y Evaluación Educativa, 21(1), 1-16.

https://doi.org/10.7203/relieve.21.1.5169

Tejada, J. (2009). Competencias docentes. Profesorado, Revista de Currículum y Formación del Profesorado, 13(2), 1-15.

Torelló, Ó. (2012). Las competencias del docente universitario: la percepción del alumno, de los expertos y del propio protagonista. REDU. Revista de Docencia Universitaria, 10(2), 299-318. https://doi.org/10.4995/redu.2012.6109

Villamizar, G., Becerra, D. y Delgado, A. (2016). Autopercepción de estudiantes de psicología sobre sus competencias en los campos laboral, educativo y salud. REXE. Revista de Estudios y Experiencias en Educación, 13(26), 151-167.

Zaragoza, J., Luis-Pascual, J. y Manrique, J. (2009). Experiencias de innovación en docencia universitaria: resultados de la aplicación de sistemas de evaluación formativa. Revista de Docencia Universitaria, 7 (4), 1-33. https://doi.org/10.4995/redu.2009.6232

\section{Cv de los autores}

\section{Francisco Javier Gallardo-Fuentes}

Profesor Asociado del departamento de Ciencias de la Actividad Física de la Universidad de los Lagos, Osorno, Chile, integrante del Núcleo de Investigación en Salud, Actividad Física y Deporte. Sus principales líneas de investigación son: la evaluación formativa y compartida en educación superior y las ciencias del deporte. ORCID ID: 0000-0002-3985157X. Email: fgallardo@ulagos.cl

\section{Bastian Carter-Thuillier}

Profesor de la Facultad de Educación, Universidad Católica de Temuco, integrante del Núcleo de Investigación en Salud, Actividad Física y Deporte de la Universidad de los Lagos. Sus principales líneas de investigación son: La evaluación de aprendizajes, El deporte y la escuela, inmigración y deporte, y Las ciencias de la actividad física. ORCID ID: 0000-0001-7342-4179. Email: bcarter@uct.cl

\section{Víctor Manuel López-Pastor}

Profesor de la Facultad de Educación de Segovia (Universidad de Valladolid). Ha publicado 20 libros y numerosos artículos científicos y profesionales. Sus principales líneas de investigación son: Evaluación Formativa en educación superior, Evaluación Formativa en educación física, Formación del Profesorado, Investigación-Acción. ORCID ID: 00000003-2681-9543. Email: vlopez@mpc.uva.es 